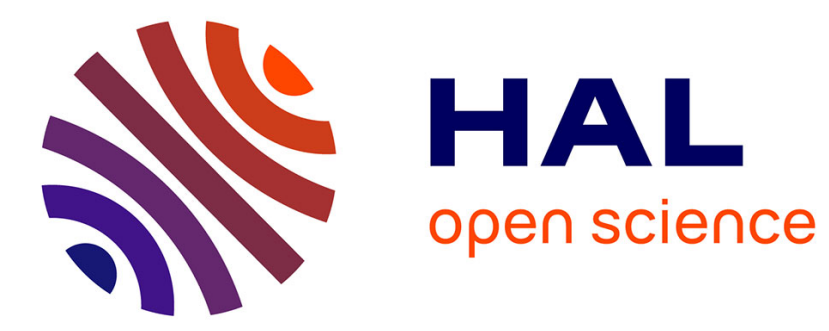

\title{
Self-consistent aspects of x-ray absorption calculations
} Oana Bunau, Yves Joly

\section{To cite this version:}

Oana Bunau, Yves Joly. Self-consistent aspects of x-ray absorption calculations. Journal of Physics:

Condensed Matter, 2009, 21, pp.345501. 10.1088/0953-8984/21/34/345501 . hal-00687301

\section{HAL Id: hal-00687301 https://hal.science/hal-00687301}

Submitted on 12 Apr 2012

HAL is a multi-disciplinary open access archive for the deposit and dissemination of scientific research documents, whether they are published or not. The documents may come from teaching and research institutions in France or abroad, or from public or private research centers.
L'archive ouverte pluridisciplinaire HAL, est destinée au dépôt et à la diffusion de documents scientifiques de niveau recherche, publiés ou non, émanant des établissements d'enseignement et de recherche français ou étrangers, des laboratoires publics ou privés. 


\title{
Self-consistent aspects of x-ray absorption calculations
}

\author{
O Bunău and Y Joly \\ Institut Néel, CNRS and Université Joseph Fourier, BP 166, F-38042 Grenoble Cedex 9 , \\ France \\ E-mail: oana.bunau@grenoble.cnrs.fr
}

Received 11 May 2009, in final form 24 June 2009

Published 5 August 2009

Online at stacks.iop.org/JPhysCM/21/345501

\begin{abstract}
We implemented a self-consistent, real-space x-ray absorption calculation within the FDMNES code. We performed the self-consistency within several schemes and identified which one is the most appropriate. We show a method that allows a rigorous setting of the Fermi level and thus an estimation of the energy cutoff for the identification and elimination of the occupied states. We investigated what are the structures where one can afford performing the self-consistent calculation at a lesser cluster radius than the absorption one. We exemplify the effects of the self-consistency at the $\mathrm{K}$-edge and for several reference cases, including the copper $\mathrm{Cu}$ and the rutile $\mathrm{TiO}_{2}$. We verified the robustness of our procedure on the transitional $3 \mathrm{~d}$ and $4 \mathrm{~d}$ elements. Although amelioration can be noticed, the self-consistency performed at the K-edge does not bring a major improvement of the calculated spectra. Taking into consideration a non-self-consistent, non-spherical potential gives better results than a self-consistent muffin-tin approximation calculation.
\end{abstract}

\section{Introduction}

X-ray near-edge absorption (XANES), as well as the related spectroscopies such as RXS (resonant X-ray scattering) and XMCD (x-ray magnetic circular dichroism), are powerful means of probing the electronic structure around the absorbing atom. In the near-edge region one is very sensitive to the surroundings of the absorbing atom. This makes absorption spectroscopies a very useful tool to investigate geometric and electronic structure.

There is a great need for codes which are able to reproduce the near-edge structure within a reasonable calculation time. Providing a satisfactory description of such complex electronic scattering phenomena is a theoretical challenge and numerous attempts have been reported.

They can be classified into two major categories. The first one is multielectronic but monoatomic, based on the multiplet theory [1]. They give a parametrized description of the localized electronic states which provides satisfactory results, but cannot describe correctly the delocalized ones. The second category is formed by the multiatomic but monoelectronic codes [2-8] which give a correct description of the extended states but fail to account for the highly correlated ones, where the interaction with the core hole is strong (e.g. the calculation of the $L_{2}$ and $L_{3}$ edges of the $3 d$ elements). This is due to the relatively long timescale associated with the localized level, as compared to the core hole lifetime. Another aspect ill-described by a monoelectronic calculation is the offset of the absorption spectra. We note that there are attempts to perform multiatomic and multielectronic x-ray absorption calculations, based on the time-dependent density functional theory [5, 9], Bethe-Salpeter equation $[5,9]$ or on the multichannel methods [10]. Nevertheless these approaches are still in their early phase and require further investigation.

The most difficult part in obtaining the absorption spectra (1) is the calculation of the final states. As far as multiatomic, monoelectronic calculations are concerned, one can do this either in the real space [2-5] or in the reciprocal one [6-8]. Both approaches give good results and are completely equivalent, provided that the cluster used for the real-space calculations and the supercell in the reciprocalspace calculations (introduced because of the breaking of periodicity due to the core hole) are large enough. In this context, performing a monoelectronic calculation means that one uses a DFT-like potential which accounts for some correlation effects.

The band structure calculations are generally selfconsistent. This is usually not the case for the realspace calculations, with the notable exception of the FEFF code $[11,12]$. The issue of self-consistency in the real-space 
calculations has not been concluded upon and needs further analysis. The purpose of this paper is to describe and to analyse the self-consistent aspects of the calculations performed with the FDMNES code [2] and compare the result with the nonself-consistent calculations.

The outline of this paper is as follows. To begin with we show some basic equations of the absorption spectroscopies. In section 2 we introduce the calculation code. In section 3 we focus on some specific points of the multiple scattering theory (MST) that are essential for our calculation. In section 4 we detail the implementation of our self-consistent scheme. In the following two sections we show our results and conclude upon the self-consistency issue at the K-edge.

\subsection{Equations of XANES and RXS}

Absorption and resonant diffraction's main featured quantities are the cross section $\sigma(\omega)$ and the structure factor $F(Q, \omega)$, both normalized with respect to the unit cell:

$$
\begin{gathered}
\sigma(\omega)=4 \pi^{2} \alpha \hbar \omega \sum_{j} \sum_{\mathrm{f}, \mathrm{g}}\left|\left\langle\psi_{\mathrm{f}}|\hat{\mathcal{O}}| \psi_{\mathrm{g}}^{(j)}\right\rangle\right|^{2} \delta\left(\hbar \omega-\left(E-E_{\mathrm{g}}^{(j)}\right)\right) \\
F(\boldsymbol{Q}, \omega) \propto \sum_{j} \mathrm{e}^{\mathrm{i} \boldsymbol{Q} \cdot \boldsymbol{R}_{j}}\left(f_{0 j}+f_{j}^{\prime}(\omega)+\mathrm{i} f_{j}^{\prime \prime}(\omega)\right)
\end{gathered}
$$

with [13]

$$
\begin{aligned}
& f_{j}^{*}(\omega) \\
& =\frac{m_{\mathrm{e}}}{\hbar^{2}} \sum_{\mathrm{f}, \mathrm{g}} \frac{\left(E-E_{\mathrm{g}}^{(j)}\right)^{2}\left\langle\Psi_{\mathrm{g}}^{(j)}\left|\hat{\mathcal{O}}_{\mathrm{out}}^{*}\right| \Psi_{\mathrm{f}}\right\rangle\left\langle\Psi_{\mathrm{f}}\left|\hat{\mathcal{O}}_{\mathrm{in}}\right| \Psi_{\mathrm{g}}^{(j)}\right\rangle}{\hbar \omega-\left(E-E_{\mathrm{g}}^{(j)}\right)+\frac{i}{2} \Gamma_{\mathrm{f}}(E)} .
\end{aligned}
$$

Here $\alpha$ is the fine structure constant, $m_{\mathrm{e}}$ the electron mass, $\hbar \omega$ the photon energy, $\delta(E)$ the electronic density of states, $E_{\mathrm{F}}$ the Fermi energy, and $E_{\mathrm{g}}$ and $E$ are the energies of the ground state $\Psi_{\mathrm{g}}^{(j)}$ and photo-excited state $\Psi_{\mathrm{f}}$, respectively. X-ray absorption requires a core, localized level as a ground state; thus we introduced the index $j$ describing the atom. The summation over $j$ adds the contributions of all the atoms in the unit cell. The final state $\Psi_{\mathrm{f}}$ is an unoccupied state of the continuum; we omit its $j$ index. $\boldsymbol{Q}$ is the diffraction vector and $\boldsymbol{R}_{j}$ is the position vector of atom $j$ in the unit cell. $\Gamma_{\mathrm{f}}$ is the sum of the inverse of the final state and the core hole lifetimes, and thus depends on the chemical type of the absorber. The atomic structure factors intervening in (2) are, respectively, the offresonant, nearly isotropic, energy-independent Thompson term $f_{0 j}$ and the complex resonant contribution $f_{j}^{\prime}(\omega)+\mathrm{i} f_{j}^{\prime \prime}(\omega)$. We agree to use a sign convention in (3) such that $f^{\prime}(\omega)<$ 0 and $f^{\prime \prime}(\omega)>0$. For the diffraction part, as we deal with a scattering process, we need to distinguish between the incoming photon field operator $\hat{\mathcal{O}}_{\text {in }}$ and the outgoing one $\hat{\mathcal{O}}_{\text {out }}$.

The electron-photon interaction is described classically by means of the field operator $\hat{\mathcal{O}}$. In the $\mathrm{x}$-ray regime, the magnetic part of the electromagnetic field can be neglected and the remaining electric part is satisfactorily described by the first two terms of the multipolar expansion, corresponding to the excitations of the electric dipole and of the electric quadrupole:

$$
\hat{\mathcal{O}}=\boldsymbol{\epsilon} \cdot \boldsymbol{r}\left(1+\frac{\mathrm{i}}{2} \boldsymbol{k} \cdot \boldsymbol{r}\right)
$$

where $r$ is the position from the absorbing ion, $\boldsymbol{\epsilon}$ the polarization of the photon and $k$ its wavevector.

\section{The FDMNES code}

The FDMNES (standing for finite difference method nearedge structure) package is an $a b$ initio, free and open source, parallelled under MPI, code which calculates the XANES, RXS and XMCD spectra. Because of their anisotropy, absorption spectroscopies are convenient to be described with a tensorial algebra. FDMNES allows a full analysis of both Cartesian and spherical tensors.

FDMNES is a real-space, symmetrized code. Whether we deal with a molecule or a periodic system, FDMNES builds a cluster around the absorbing atom. The cluster's radius needs to be large enough in order to achieve convergence with respect to the accuracy of the calculation of the final states.

\subsection{The procedure}

In this paragraph we will detail the steps of the calculation. We start by performing a self-consistent atomic calculation in order to get the initial level $\Psi_{\mathrm{g}}$ for the absorber and the atomic densities for the atoms in the cluster. To calculate the absorption signal, we assume an excited electronic configuration for the absorbing atom, i.e. we place the core electron on the first available unoccupied level. We superpose the atomic densities to get the charge density of the cluster and we solve the Poisson equation to get the Coulomb potential. We improve this potential by considering an energy-dependent exchange-correlation correction that one can choose among the Perdew and Wang [14] and the real Hedin-Lundquist $[15,16]$ potentials. Once the potential has been constructed we solve the Schrödinger-like equation (SE) to get the final states $\Psi_{\mathrm{f}}(\boldsymbol{r})$ (or some related quantities). Next we calculate the matrix elements featured by equations (1) and (3). Finally we perform the sum over the states in (1) and (3) and the convolution.

\subsection{The Schrödinger-like equation}

FDMNES features two ways of calculating $\Psi_{\mathrm{f}}(r)$ : the finite differences method (FDM) and the multiple scattering theory (MST), within the limits of the muffin-tin approximation (MT). The latter assumes a crystal potential that has spherical symmetry around the atoms (the so-called muffin-tin spheres) and is constant in the remaining space. A radial SE is solved within each sphere. The former uses the full potential and consists in constructing a space grid and discretizing the SE on the points of this grid. FDM does not introduce any approximation on the form of the potential and thus provides a more accurate but more time-consuming description of the scattering phenomena.

\subsection{The convolution}

The spectrum in (1) requires the convolution with a Lorentzian having an energy-dependent width $\Gamma_{\mathrm{f}}(\omega)$ in order to account for the broadening due both to the core hole width $\Gamma_{\text {hole }}$ and to the spectral width $\gamma(\omega)$ of the final state:

$$
\sigma^{\text {conv }}(\omega)=\int_{E_{\mathrm{F}}}^{\infty} \mathrm{d} E \sigma^{\text {nonconv }}(E) \frac{1}{\pi} \frac{\Gamma_{\mathrm{f}}(\omega)}{\Gamma_{\mathrm{f}}(\omega)^{2}+(\hbar \omega-E)^{2}}
$$




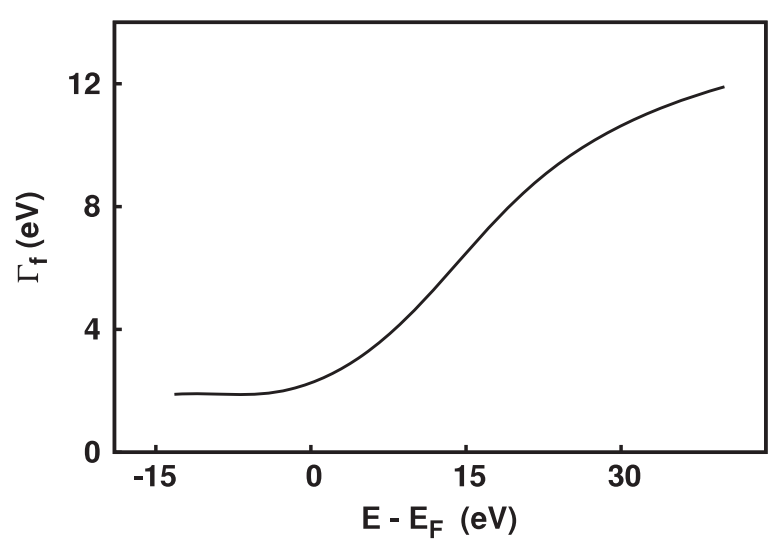

Figure 1. $\Gamma_{\mathrm{f}}$ has an arctangent-like form, centred at $E_{\mathrm{ctr}}$ and whose defining parameters are the slope $\Gamma_{\max } / E_{l}$ at $E_{\mathrm{ctr}}$ and the total height $\Gamma_{\max }$.

with

$$
\Gamma_{\mathrm{f}}(\omega)=\Gamma_{\text {hole }}+\gamma(\omega) .
$$

Here $\sigma^{\text {nonconv }}(\omega)$ and $\sigma^{\text {conv }}(\omega)$ are the unconvoluted and the convoluted absorption signal, respectively. In practise, one uses the standard, tabulated [17], edge-dependent and energyindependent (i.e. the screening is treated statically) values for $\Gamma_{\text {hole }}$. The energy dependence of the broadening is entirely due to $\gamma$ and is a signature of the inelastic electron scattering phenomena.

We agree to set $\gamma$ according to the arctangent model, an empirical model close to the Seah-Dench formalism [18]:

$$
\begin{aligned}
& \Gamma_{\mathrm{f}}\left(E-E_{\mathrm{F}}\right)=\Gamma_{\text {hole }}+\Gamma_{\max } \\
& \times\left(\frac{1}{2}+\frac{1}{\pi} \arctan \left(\frac{\pi}{3} \frac{\Gamma_{\max }}{E_{l}}\left(e-\frac{1}{e^{2}}\right)\right)\right) ; \\
& e=\frac{E-E_{\mathrm{F}}}{E_{\mathrm{ctr}}}
\end{aligned}
$$

where $E_{\mathrm{F}}$ is the Fermi energy and the parameters describing the arctangent are: the total height $\Gamma_{\max }=15 \mathrm{eV}$, the inflection point $E_{\mathrm{ctr}}-E_{\mathrm{F}}=30 \mathrm{eV}$ and the inclination $E_{l}=30 \mathrm{eV}$ of the arctangent (figure 1). As far as the calculations in this paper are concerned, we maintained these parameters unchanged. In figure 1 , the inflection point corresponds to the emergence of the plasmons. The energy scale is referenced to the Fermi level $E_{\mathrm{F}}$ whereas its zero points to vacuum energy. To have an approximate indication on the energy of the photon, one should add the energy of the edge.

We use the same $\Gamma_{\mathrm{f}}$ as in the sum over the states for the structural factor calculation in (3). Contrary to the convolution of the spectrum in (1), the convergence of (3) over the energies is extremely slow. To overcome this problem, one uses the imaginary part of $f(\omega)$ to the atomic form factor up to $10000 \mathrm{eV}$ above the highest calculated energy.

In the (1) and (3) summations over the states, only unoccupied states appear. Consequently, one must eliminate the contribution of all $\Psi_{\mathrm{g}}^{(j)}$ corresponding to states whose energies are below the Fermi energy. The sum over the final states in (1) becomes

$$
\sum \equiv \int_{E_{\mathrm{F}}}^{\infty} \mathrm{d} E \sum_{\mathrm{f}}
$$

with $\mathrm{f}$ being the final states of the same energy $E$ (normalized at one state per Rydberg). In some cases the lower limit of this integral is different from the Fermi energy $E_{\mathrm{F}}$, as we shall discuss in section 5.1 .

\section{The multiple scattering theory}

In this paper we will focus on the MST as an alternative to solve the SE for an electron moving in a potential $\hat{V}$. We show the link with the electronic density, which we use in our self-consistent procedure. Only non-magnetic cases are considered; we therefore make no further reference to the electron spin. In this approach we calculate the multiple scattering amplitude matrix $\tau_{l, m}^{l^{\prime} m^{\prime} ;(j)}(E)$, also known as the transmission $t$ matrix, for each atomic scatterer $j$ and projected on the spherical harmonics basis. As the $t$ matrix is related to the full Green function $\hat{\mathcal{G}}$, we thus have a complete description of the interacting system [19]:

$$
\mathcal{G}\left(\boldsymbol{r}, \boldsymbol{r}^{\prime} ; E\right)=\sum_{l, l^{\prime}} \sum_{m, m^{\prime}} \Psi_{l^{\prime}, m^{\prime}}^{*}\left(\boldsymbol{r}^{\prime} ; E\right) \tau_{l, m}^{l^{\prime} m^{\prime} ;(j)}(E) \Psi_{l, m}(\boldsymbol{r} ; E) .
$$

Equation (9) is valid in the interior of the muffin-tin sphere for the $j$ scatterer, where the wavefunction describing the final states can be expanded in the spherical harmonics basis.

We use the expansion

$$
\Psi_{\mathrm{f}}(\boldsymbol{r} ; E)=\sum_{l, m} a_{l, m}^{\mathrm{f}}(E) b_{l}(r, E) Y_{l}^{m}(\hat{r}) .
$$

Here $Y_{l}^{m}(\hat{r})$ are the spherical harmonics (which we can take in either the real or the complex basis, depending on the symmetry) and $\phi_{l}(r ; E)=r b_{l}(r, E)$ is the solution of the radial SE at energy $E . \quad a_{l, m}^{\mathrm{f}}(E)$ are the energydependent atomic amplitudes chosen to ensure the continuity of the solution in the muffin-tin sphere with the free-space wavefunction. The latter is normalized to the density of states of the continuum, hence $\Psi_{\mathrm{f}}(\boldsymbol{r} ; E)$ has the adequate norm. In practise, we cut this expansion at a maximum value $l_{\max }+1$ of the $l$ quantum number, which depends on the energy according to the rule

$$
\sqrt{l_{\max }\left(l_{\max }+1\right)}=\sqrt{E} r_{\mathrm{MT}}
$$

where both the energy $E$ and the muffin-tin radius $r_{\mathrm{MT}}$ are expressed in atomic units.

We briefly recall the mechanism of the multiple scattering: the absorbing atom receives both the incoming wave $\Psi_{0}$, i.e. a term of the expansion in (10), and those scattered by the atomic neighbourhood. One needs to have a cluster large enough to get the accurate final states $\Psi_{\mathrm{f}}$. The core state being fixed, the energy dependence of the scattering lies in the photo-excited state $\Psi_{\mathrm{f}}$. Hence the density operator can be written as

$$
\hat{\rho}=\int_{-\infty}^{E_{\mathrm{F}}} \mathrm{d} E \sum_{\mathrm{f}}\left|\Psi_{\mathrm{f}}\right\rangle\left\langle\Psi_{\mathrm{f}}\right|
$$

with $\mathrm{f}$ being the final states of the same energy $E$ and $E_{\mathrm{F}}$ the Fermi energy. 
The multiple scattering matrix element $\tau_{l, m}^{l^{\prime} m^{\prime}}(E)$ is calculated using the atomic scattering amplitudes $a^{\mathrm{f}}(E)$ via the optical theorem (13).

In the following paragraphs we will show the form of the optical theorem, as well as the density-related quantities (density of states $\delta(E)$, density of particles $\rho(\boldsymbol{r})$, occupation number $N$ ) in the limit of a zero spin-orbit coupling.

When the potential is real, the optical theorem gives

$$
\left|\sum_{\mathrm{f}} a_{l^{\prime} m^{\prime}}^{\mathrm{f} *}(E) a_{l, m}^{\mathrm{f}}(E)\right|=-\operatorname{Im}\left(\tau_{l, m}^{l^{\prime} m^{\prime}}(E)\right)
$$

with $\mathrm{f}$ being the final states of the same energy $E$.

The density of states $\delta(E)$ of an interacting system is given by the imaginary part of its associated Green operator $\hat{\mathcal{G}}(E)$. We can express all the density-related quantities (density of states, charge density, occupation number, etc) in terms of the MST matrix elements $\tau_{l, m}^{l^{\prime} m^{\prime}}(E)$ and of the radial solutions $b_{l, m}(r, E)$.

For an atom, we use (10) and (12) to get the density operator matrix element in the representation of spherical harmonics:

$$
\begin{aligned}
& \frac{\mathrm{d}}{\mathrm{d} E} \rho_{l, m}^{l^{\prime} m^{\prime}}(r, E)=b_{l^{\prime}}^{*}(r, E) b_{l}(r, E) \\
& \quad \times \sum_{\mathrm{f}} a_{l^{\prime}, m^{\prime}}^{\mathrm{f} *}(E) a_{l, m}^{\mathrm{f}}(E) \\
& \rho_{l, m}^{l^{\prime} m^{\prime}}(r)=-\operatorname{Im} \int_{-\infty}^{E_{\mathrm{F}}} \mathrm{d} E b_{l^{\prime}}(r, E) \tau_{l, m}^{l^{\prime} m^{\prime}}(E) b_{l}(r, E)
\end{aligned}
$$

where the integral is taken over all the energies under the Fermi level $E_{\mathrm{F}}$ and $\mathrm{f}$ are the final states of the same energy $E$. The electronic density of states $\delta(E)$ is calculated similarly:

$$
\delta(E)=-\operatorname{Im} \int \mathrm{d}^{3} r \sum_{l, m} b_{l}(r, E) \tau_{l, m}^{l, m}(E) b_{l}(r, E) .
$$

The expectation value of the occupation number is obtained by taking the trace of the $\hat{\rho}$ matrix, i.e.

$$
N=\int_{-\infty}^{E_{\mathrm{F}}} \mathrm{d} E \delta(E)=\int \mathrm{d}^{3} r \sum_{l, m} \rho_{l, m}^{l, m}(r) .
$$

\subsection{The scattering in a complex potential}

When the potential is complex the radial SE has two admissible solutions, a regular and an irregular one, according to the behaviour of $\Phi_{l}(r)=r u_{l}(r)$ in the origin $r=0$. One has to account for both of them when writing the Green's function. In the absence of the spin-orbit coupling it has been shown [20] that

$$
\begin{gathered}
\mathcal{G}^{+}\left(\boldsymbol{r}, \boldsymbol{r}^{\prime} ; E\right)=\sum_{l, m} \sum_{l^{\prime}, m^{\prime}} b_{l^{\prime}}\left(r^{\prime}, E\right) b_{l}(r, E) Y_{l^{\prime}}^{m^{\prime}} Y_{l}^{m} \\
\times\left(\tau_{l, m}^{l^{\prime}, m^{\prime}}(E)-t_{l, m}^{l^{\prime}, m^{\prime}}(E)\right)-\sum_{l, m} t_{l, m}^{l, m}(E) \\
\times b_{l}\left(r_{<}, E\right) b_{l}\left(r_{>}, E\right) Y_{l}^{m} Y_{l}^{m}
\end{gathered}
$$

where $r_{<}=\min \left(r, r^{\prime}\right)$ and $r_{>}=\max \left(r, r^{\prime}\right)$. We can easily get the density of states by taking the trace of the $\mathcal{G}^{+}$matrix:

$$
\delta(E)=-\operatorname{Im} \int \mathrm{d}^{3} r \mathcal{G}^{+}(\boldsymbol{r}, \boldsymbol{r} ; E) .
$$

The $\hat{\rho}$ matrix becomes

$$
\begin{aligned}
\frac{\mathrm{d}}{\mathrm{d} E} & \rho_{l, m}^{l^{\prime}, m^{\prime}}(r, E)=-\operatorname{Im}\left[\left(\tau_{l, m}^{l^{\prime}, m^{\prime}}(E)-t_{l, m}^{l^{\prime}, m^{\prime}}(E)\right)\right. \\
& \times b_{l^{\prime}}(r, E) b_{l}(r, E)+\delta_{l l^{\prime}} \delta_{m m^{\prime}} \\
& \left.\times b_{l}(r, E) t_{l, m}^{l, m}(E) b_{l}(r, E)\right] .
\end{aligned}
$$

To end with, we shall try to provide a brief insight into the physical meaning of a complex potential one shall further use in the implementation of the self-consistent scheme. Numerically, one can prove that performing the convolution of (5) on the output of a real potential calculation is identical to the unconvoluted result for the equivalent complex potential calculation (i.e. the imaginary part of the potential is equal to the $\Gamma_{\mathrm{f}}$ broadening of the former) in the limit $E_{\mathrm{F}} \rightarrow-\infty$. Therefore the use of a complex potential is synonymous with taking into account some inelastic photo-electron scattering mechanisms.

\section{Implementation of the self-consistency}

We introduced the self-consistency within the multiple scattering frame. By self-consistency we mean that a first calculation is performed and that the resulting electronic density is used to derive the potential used during the next iteration. This procedure is repeated until convergence is achieved. Once we are sure to have the correct electronic levels, we perform a last iteration which calculates the absorption spectra (1), in either the MST or the FDM approach. We note that the cluster's radius for this last calculation is not necessarily the same as the one used at the previous iterations; in particular we can be interested in running an absorption calculation at a larger radius than the one used in order to get the electronic density in a self-consistent manner. In the following we will discuss the implementation of the selfconsistent scheme.

We distinguish between three ways of performing the self-consistent procedure, which we will further reference by (a), (b) and (c), respectively. Scheme (a) assumes a nonexcited electronic configuration for the absorber during the entire calculation. The second possibility (b) is to assume an excited absorber, i.e. in the presence of a core hole and with an extra electron on the first available valence level. Finally, there is a third scheme (c) corresponding to an intermediate situation between (a) and (b): the self-consistency is performed on a non-excited atom, in order to get the accurate final states $\psi_{\mathrm{f}}$. It is only during the absorption calculation that we take into account the excited electronic structure of the absorber. In practise, at the beginning of the absorption calculation, we add the difference between the atomic density corresponding to an excited atom and the one for the non-excited case to the self-consistent electronic density of the absorber. We note that both in (b) and in (c) we introduce by default a full screening to calculate absorption. The multielectronic effects due to the core hole are taken into account through the final state rule [21, 22]. The essential dissimilarity between the (b) and (c) procedures is linked to the difference in the timescales associated with the two main processes involved: the passage of the photo-electron and the response of the 
electron cloud surrounding the absorber. The electrons either adjust instantaneously to the perturbed structure, in which case scheme (b) is appropriate, or their reactivity is latent with respect to the advent of the photo-electron, in which case scheme (c) is more accurate. In the next section we shall validate the most accurate procedure among the three.

We cycle on a wide range of electron energies (we set an energy step of $0.1 \mathrm{eV}$ ) and we calculate the $a(E)$ and $b_{l}(r, E)$ factors for each of these energies. The first energy of the calculation is chosen between those of the last core orbital and of the first valence one, in terms of the atomic energy levels of the atoms in the cluster. We define the core states as those atomic orbitals which are part of a full shell and are far enough from the potentially occupied states; the remainder are referred to as valence states. We recall that the atomic levels have no physical significance if the cluster contains several atoms; they still give us a fair indication of where the calculation should begin, in order not to miss the electrons we are interested in. We note that beginning the calculation at a rather low energy (at around $30-80 \mathrm{eV}$ below the edge) is not very time-consuming, because of the small number of spherical harmonics featured in the expansion, according to the rule in (11).

Calculation time is saved by means of the symmetrization. All calculations are symmetrized, but different symmetry constraints are applied according to the choice of the selfconsistent scheme ((a), (b) or (c)). Procedure (a) is consistent with the periodic description of a crystal. In this case we calculate electronic densities corresponding to the nonequivalent atoms in the unit cell and impose them to the rest of the atoms in the cluster, according to the space group symmetry. This scheme avoids the convergence problems due to border phenomena and concerning the atoms far from the absorber. Within scheme (b), when we deal with an excited absorber, the crystal symmetry is broken by the presence of the core hole which renders the calculation molecular-like. The space group symmetry is no longer relevant and we need to calculate the electronic structure of atoms independently, at most by taking into account the point group symmetry of the absorber. One straightforward consequence of this procedure is the fact that it induces artificial effects at the cluster's borders. The lack of constraints with regard to the distribution of electrons determines them to accumulate at the borders. This phenomenon does not prevent convergence at the K-edge.

In practise, in order to calculate the potential for the atoms at the border of the cluster, we use an auxiliary calculation cluster, whose radius is larger than the former's. The additional atoms it brings are not calculated self-consistently. Their only purpose is to set the atomic levels used for the superposition of both the electronic densities and the atomic potentials.

The energies of the calculation are chosen to have a small $(0.1 \mathrm{eV})$ imaginary part. This is needed in order to broaden the localized electronic levels, otherwise we would need an extremely small energy step to count properly the electrons in these orbitals. We therefore need to refer to equations (18) and (20) for the calculation of all the density-related quantities. Given the fact that the imaginary part of the complex potential is typically one or two orders of magnitude smaller than the parameters defining the convolution function (section 2.3), one should not be bothered by the fact that the spectral broadening in (5) is taken into account twice.

The evaluation of the Fermi level demands the setting of a reference in terms of the number of valence electrons one can find in the cluster. Summing up the atomic numbers is not exact, for two reasons. Firstly, we count only the electrons lying within the atomic spheres $R_{j}$, whose construction is explained in the following paragraph. Secondly, we fail to count some valence electrons localized beyond the cluster's borders and which belong to the next-to-the-border atoms.

To solve this, we assign a specific space extension to each atom. We consider spherical atoms $j$ with radii $R_{j}$, chosen in such a way that the cluster is neutral. This procedure has been proved inappropriate for certain kinds of materials (i.e. sparse structures) as it implies too great of an overlap between the atomic spheres. In this case we set an upper limit of $30 \%$ of overlap with respect to the sum of the volumes of the spheres in the unit cell. We note that the atomic radii are usually larger than the muffin-tin radii. One calculates the spatial integrals in the following manner:

$$
\int_{\text {space }} \mathrm{d}^{3} r=\sum_{j} \int_{0}^{R_{j}} \mathrm{~d}^{3} r
$$

by summing the relevant atomic sphere over all the atoms of the cluster.

Subsequently, we use (17) and (21) to calculate the number of electrons belonging to each atom and then the total electronic population of the cluster. The first calculation is stopped when this number reaches the reference electron number. We get the corresponding electron charge density. In practise, as usual, we perform a weighting of the charge density at the previous iteration (that we used at the beginning of the cycle in order to calculate the Coulomb potential) with the current one. The experience tells us that the current calculation needs to have a rather small weight ( 0.1 at most) for convergence to be achieved. The interpolation parameter is set up dynamically, i.e. we decrease it by a factor of 2 in case of a beating convergence parameter, which we define later in (23). We inject the weighted charge density into the next cycle and we repeat the calculation until the convergence is achieved.

We note that the automatic setting of the Fermi level is a very convenient and user-friendly feature of the self-consistent calculation. For the codes where this is not the case, the Fermi level is set ad hoc and thus the elimination of occupied states according to (8) is spurious. In the case of sparse structures (as one will see in section 5) the calculated Fermi level and the cutoff of the absorption spectrum may differ; nevertheless the former still gives valuable information (within $\approx 1-5 \mathrm{eV}$ ).

The total energy of the cluster $\mathcal{E}$ is calculated by integrating the multiple scattering calculated quantities into the Kohn-Sham formula [23]:

$$
\begin{aligned}
\mathcal{E}[\rho(r)] & =\sum_{\text {atoms }} \int \mathrm{d} E E \delta(E)+\sum_{\text {atoms }} \sum_{g \in \text { core }} \epsilon_{\mathrm{g}} \\
& -\int \mathrm{d}^{3} r \rho(r) V_{\mathrm{c}}(\rho(r))+\int \mathrm{d}^{3} r \rho(r) \epsilon_{\mathrm{xc}}(\rho(r)) \\
& -\int \mathrm{d}^{3} r \rho(r) V_{\mathrm{xc}}(\rho(r))
\end{aligned}
$$


where $f$ are the final states of the same energy $E$. The first term of (22) represents the sum over the occupied valence orbitals of the eigenvalues we got by solving the SE. $V_{\mathrm{c}}$ is the bare Coulomb potential, $V_{\mathrm{xc}}$ is the exchange-correlation potential and $\epsilon_{\mathrm{xc}}$ is the exchange correlation energy. The second term, which we do not calculate explicitly, is the energy of the core states, calculated in the monoelectronic picture. What we calculate is the variation of this energy with respect to the first iteration value, under the assumption that this variation is entirely due to the changing potential and that the core wavefunction is the atomic one. Consequently, the energy in (22) is defined up to an additive constant.

The criterion to achieve convergence is to have a stable total cluster energy $\mathcal{E}$, i.e. the variation of this quantity from one iteration to the next must be inferior to a particular user-chosen value. In practise, we take into account the less favourable situation where the variations of energies of different atoms have different signs, from one iteration to the next. Hence we use a more severe criterion, as compared to the stability of $\mathcal{E}$ :

$$
\Delta \mathcal{E}=\sum_{\mathrm{eq}}^{p} \mathcal{N}_{p}\left|E_{p}^{i}-E_{p}^{i-1}\right|<1 \mathrm{eV} \times \sum_{p} N_{p}
$$

where we sum on all the equivalent atoms $p$, with $\mathcal{N}_{p}$ being their multiplicity and $N=\sum_{p} N_{p}$ the total number of atoms in the cluster. $E_{p}^{i}$ is the energy of such a prototype atom $p$ at the $i$ iteration. Once convergence is achieved we expect that $\mathcal{E}$ reaches its minimum amongst all the iteration values.

\section{Results}

In this section we aim to answer several questions linked to the pertinence of applying a self-consistent procedure to an $\mathrm{x}$-ray absorption calculation. In particular, we are interested to detect what are the structures where a self-consistent calculation can improve the non-self-consistent results. Another issue is whether one could afford a smaller calculation radius for the self-consistent part than for the absorption calculation, for the purpose of saving computation time. Moreover, we wish to indicate what is the best self-consistent approach among the ones presented in the previous section. To begin with, we shall study two textbook cases, the copper $\mathrm{Cu}$ and the rutile $\mathrm{TiO}_{2}$. We also discuss the results of calculations run on boron nitrate $\mathrm{BN}$ and calcium oxide $\mathrm{CaO}$, as well as on the $3 \mathrm{~d}$ elements.

\subsection{The copper $\mathrm{Cu}$}

To begin with, we will show the self-consistent calculations run on copper, as a reference case. We show the absorption spectra at the K-edge for several radii of calculation, each of them corresponding to an additional shell of atoms. Figure 2 shows the absorption spectra, calculated within the MST frame, before and after the convolution. One should always appeal to the convoluted spectra when comparing to experiment.

One can see that, in terms of calculation radii, convergence is achieved at $6.76 \AA$ (i.e. 135 atoms). For this radius

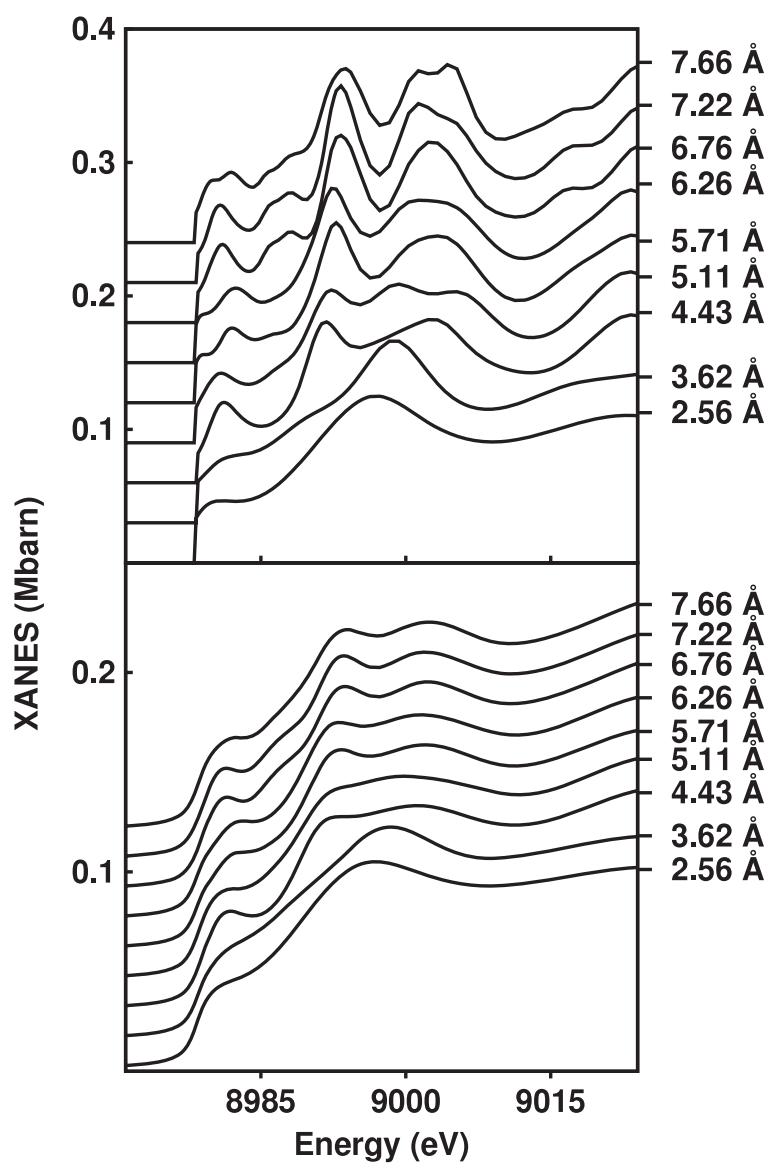

Figure 2. Evolution of the absorption signal with respect to the cluster size: before (top) and after convolution (bottom). The cutoff in the unconvoluted spectra is a consequence of the elimination of the occupied states.

we superpose the spectra after convolution for several selfconsistent and for a non-self-consistent calculation. The selfconsistent calculations have been performed in several manners ((a), (b) and (c)), according to the procedures described above (see section 4). Among these, one can notice that procedure (c) is the most appropriate with respect to the agreement with the experiments [24], as it improves the ratio of intensities of the structures after the edge. Unlike scheme (b), non-excited self-consistent procedures (a) and (c) do not influence the position of the structures preceding the white line, as compared both to the non-self-consistent case and to the experiment. We therefore conclude that the assumption we had made to get the (b) spectrum (i.e. the response of the electron cloud surrounding the absorber to the passage of the photo-electron is adiabatic) is not valid at the $\mathrm{K}$-edge, as confirmed by our further examples.

We shall discuss the issue whether the self-consistency (i.e. all but the last iteration) has to be performed at the same radius as the absorption (i.e. the last iteration) calculation. We ran a calculation where we used a cluster of $2.56 \AA$ radius (13 atoms, i.e. the absorber surrounded by the first shell of copper atoms) for the self-consistency, while the absorption calculation was performed on a cluster of $6.76 \AA$. We compared the result with the one we had previously obtained 


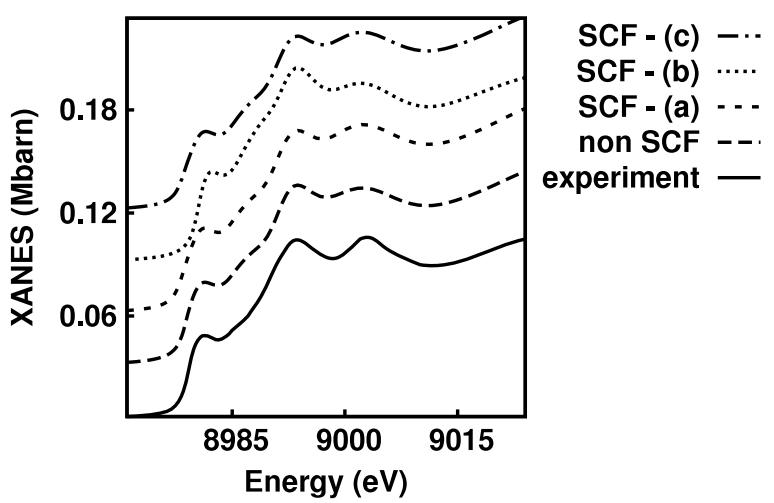

Figure 3. Calculation and experimental data for absorption at copper's K-edge for a $6.76 \AA$ cluster. As far as self-consistent calculations are concerned, we tested the three different procedures: full non-excited calculation (a), full excited calculation (b) and hybrid (c), i.e. self-consistency is performed on the non-excited absorber while the absorption takes into consideration the excited electronic structure.

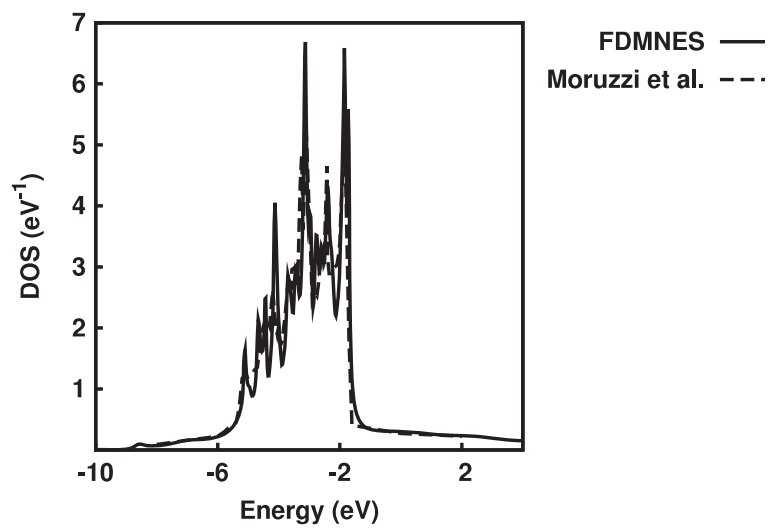

Figure 4. A comparison between the total density of states of copper calculated by FDMNES (an SCF- (a) procedure calculation) and the results of Moruzzi et al [25]. The zero of the energy scale corresponds to the Fermi level [25].

(figure 3) for a full $6.76 \AA$ A calculation. We found practically no difference between the two, which encourages us to use a smaller cluster for the self-consistency part for the copper-like (i.e. compact) structures.

Finally, we wish to validate our self-consistent procedure by comparing it to an equivalent band structure calculation. We chose to compare with the results of Moruzzi et al [25], who used the self-consistent, reciprocal space KKR method, leaning on the muffin-tin (MT) approximation. Our self-consistent scheme (a) is completely equivalent to a band structure result, as the absorber is supposed non-excited throughout the calculation. To ensure a high resolution, we performed a calculation on a radius of $8.47 \AA$ ( 225 atoms) taking the value of $0.025 \mathrm{eV}$ both for the energy step and for the imaginary energy. In figure 4 we compare the total density of states (DOS) for the two calculations. One can see a very good agreement of the two, which testifies of the accuracy of our self-consistent scheme.

To conclude, one may state that the self-consistency does not have a major influence in the case of copper at the $\mathrm{K}$ -

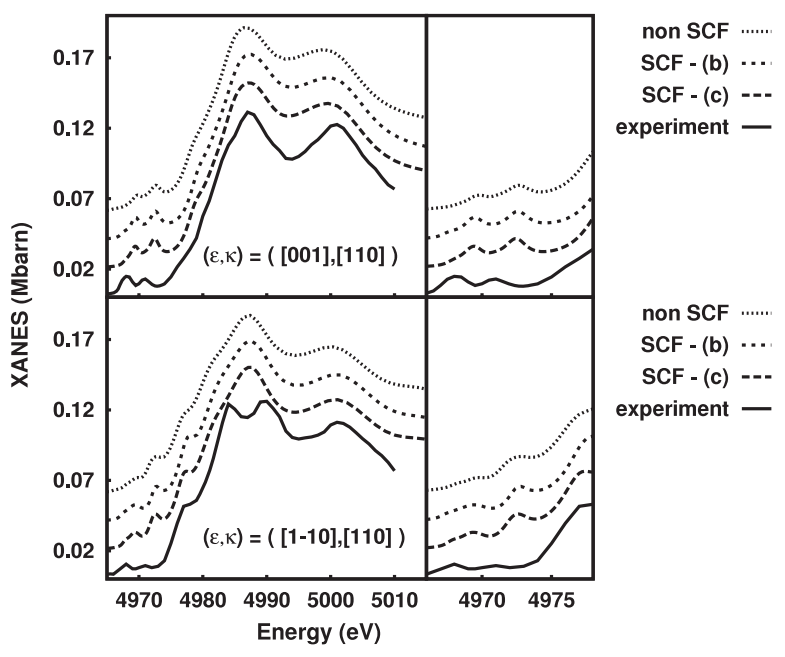

Figure 5. MST-MT calculations and experimental data [24] for absorption at titanium's K-edge for a $5.51 \AA$ cluster. Two polarizations are shown: $(\epsilon, k)=([001],[110])$ (top) and $(\epsilon, k)=([1 \overline{1} 0],[110])$ (bottom). As far as self-consistent calculations are concerned, we tested two different procedures: the full excited (b) and the hybrid (c) calculation. On the right-hand side, we show a zoom on the pre-edge structures. One can see that, compared to the non-self-consistent ones, self-consistent procedures reduce the disagreement with the experiment.

edge. This result is general for the compact, metallic structures (i.e. with a weak overlap between the atomic spheres $R_{j}$ ), as shall follow further on in the present paper. Moreover, one can afford to perform the self-consistent calculation at a smaller radius than the absorption one. The self-consistent calculation provides the Fermi level, which in practise is very useful information and is eventually one of the most important results of the self-consistency.

\subsection{The rutile $\mathrm{TiO}_{2}$}

In this section we will show the results of calculations run on the $\mathrm{TiO}_{2}$ compound. We calculate the absorption signal at the titanium's K-edge for two different polarizations. $\mathrm{TiO}_{2}$ is an interesting structure to test our self-consistent procedure on, as it features several pre-edge peaks of both dipolar and quadrupolar origin and related to the $3 \mathrm{~d}$ states of titanium. The MST non-self-consistent calculations do not give a very good agreement with the experiments. A priori one would expect benefits from a self-consistent calculation, as it is supposed to act especially on the localized $3 \mathrm{~d}$ states in the vicinity of the Fermi level.

In figure 5 we show the non-self-consistent and the self-consistent calculations (according to the two different procedures (b) and (c)) run on a $5.51 \AA$ A cluster and compare them to the experimental data of Poumellec et al [24]. We note that a radius of $5.51 \AA$ corresponds to 75 atoms and is enough to achieve convergence of the absorption signal with respect to the cluster size. We note that a condition sine qua non to obtain the pre-edge structures is that the cluster contains the first shell of $\mathrm{TiO}_{2}$ octahedra surrounding the absorber, which is achieved for the $5.51 \AA$ radius. All absorption calculations have been performed in the MST frame. We analyse the signal 


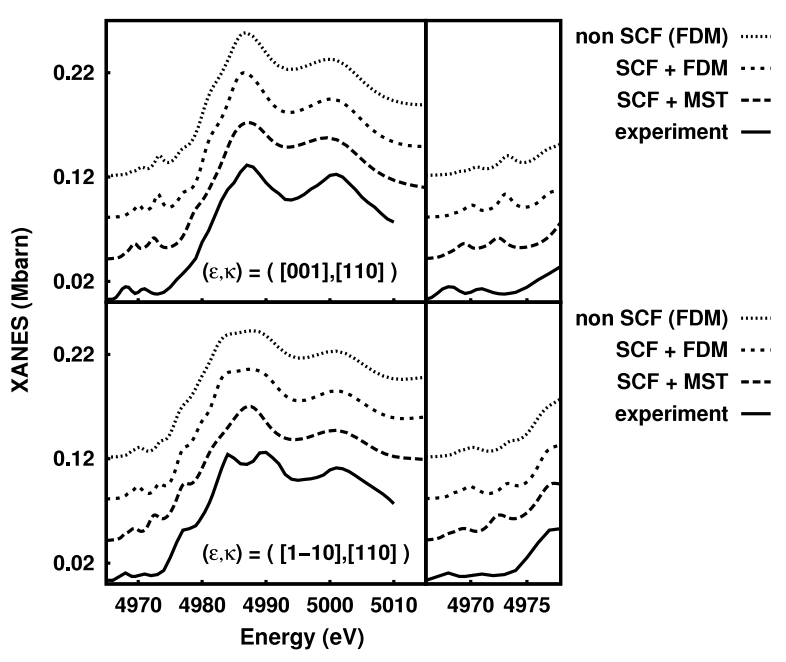

Figure 6. FDM calculations and experimental data [24] for absorption at titanium's K-edge for a $5.51 \AA$ cluster. Two polarizations are shown: $(\epsilon, k)=([001],[110])$ (top) and $(\epsilon, k)=([1 \overline{1} 0],[110])$ (bottom). The self-consistent calculations are performed according to scheme (c). On the right-hand side we show a zoom on the pre-edge structures. One can see that, compared to the non-self-consistent ones, self-consistent procedures reduce the disagreement with the experiment.

obtained for two different orientations of the polarization and the wavevector: $(\epsilon, k)=([001],[110])$ and $(\epsilon, k)=$ ([110], [110]). One can see that all calculations fail to describe the double structure at the edge (4980-4990 eV) for the second polarization. This is a defect of the MT approximation. As far as the pre-edge structures are concerned, they are due to the overlap between the absorber's electronic states and the $e_{g}$ and the $\mathrm{t}_{2 \mathrm{~g}}$ of the titanium neighbours. As compared to the nonself-consistent calculation, both procedures (b) and (c) succeed in shifting the pre-edge structures towards the smaller energies; nevertheless this shift is not enough to give a perfect agreement with the experiments. One should note that $\mathrm{TiO}_{2}$ is a rather sparse material and the MST-MT is known not to give very good results for this particular kind of structure because of the approximations it introduces on the form of the potential.

As far as the self-consistent calculations are concerned, we checked that the converged result is the same if we choose different departure points in terms of the atomic electronic structure of the absorber (i.e. $3 \mathrm{~d} 14 \mathrm{~s} 24 \mathrm{p} 1$ or $3 \mathrm{~d} 24 \mathrm{~s} 24 \mathrm{p} 0$ ), proving the robustness of our procedure. Moreover, in practise the result is nearly independent of the exchange correlation potential we used (i.e. Hedin-Lundquist $[15,16]$ or Perdew and Wang [14]).

Another aspect to take into consideration when performing the self-consistent procedure is the evolution of the atomic charges during the iterations. We recall that we set the atomic radii $R_{j}$ in order to ensure the neutrality of the unitary cell or, equivalently, of the calculation cluster. We checked that, when convergence is achieved, the modulus of the individual atom charges decreases as compared to its value at the first iteration. In the particular case of $\mathrm{TiO}_{2}$, by using the selfconsistent scheme (c) we converge to negatively charged oxygen and positively charged titanium, which is consistent with

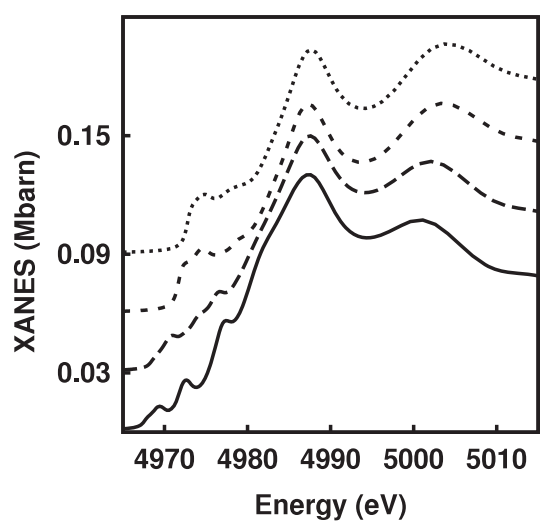

$4.60 \AA$ A...

$4.10 \AA$

$3.60 \AA--$.

$5.51 \AA$

Figure 7. Self-consistent calculations of type (c), run for different sizes of the cluster. The absorption calculation is performed on a $5.51 \AA$ cluster in the MST-MT frame.

the electrochemical picture. When convergence is achieved, we get an additional $+0.7 e$ for the titanium and $-0.35 e$ for the oxygen, with $e$ being the modulus of the electron charge. At this point, the calculated atomic charges $(0.256 e$ for the titanium and $-0.128 e$ for the oxygen) are still very different from the formal ones ( $4 e$ and $-2 e$, respectively). This is partially due to the fact that the atomic radii $R_{j}$ we used for the charge calculation (1.42 and $1.04 \AA$ ) are different from the ionic radii (0.86 and $1.4 \AA)$.

In figure 6 we show a self-consistent calculation with the absorption calculation performed in the FDM frame. The main difference between the FDM and the MST-MT calculations occurs at the edge $(4980-4990 \mathrm{eV})$, as the former is able to reproduce the double structure for the $(\epsilon, k)=([1 \overline{1} 0],[110])$ polarization. This is a consequence of having considered the full potential. As compared to its non-self-consistent counterpart, the self-consistent FDM calculation shifts the quadrupolar signal towards the lower energies. Moreover, the ratio of the intensities of the pre-edge structures is improved. The self-consistent scheme is still performed in the MST-MT frame; to get a better agreement with the experiments one should implement a full potential self-consistent calculation.

We have studied the influence of the cluster's size for the self-consistent part for a given radius $(5.51 \AA$ ) of the absorption calculation. We note that all the radii we tested are large enough to exhibit the pre-edge structures. Unlike the copper case, the results are very sensitive to the radius for the selfconsistent part (figure 7). We therefore conclude that for oxides, whose structure is sparser, one should take the same cluster size for the self-consistent part as for the absorption calculation.

\subsection{Other examples}

We tested our procedure on numerous materials for which the self-consistency brings little or no change with respect to its non-self-consistent equivalent. We show the results on both simple ( $3 \mathrm{~d}$ and $4 \mathrm{~d}$ metals) and complex elements (boron nitrate $\mathrm{BN}$ and calcium oxide $\mathrm{CaO}$ ).

We ran calculations on the $3 \mathrm{~d}$ and $4 \mathrm{~d}$ series by using the standard crystallographic structure. We encountered no 
particular difficulty, either to achieve convergence, or to obtain the correct cutoff for the convolution and, thus, the elimination of the occupied states. We chose to show the results we got for titanium, nickel, iron and silver (figure 8) and compare them to the experiment [26]. In the cases where the (b) and (c) selfconsistent schemes give different results ( $\mathrm{Ag}, \mathrm{Ni}$ and $\mathrm{Cu}$ ), the best agreement is achieved by using the former procedure. In other cases ( $\mathrm{Fe}$ and $\mathrm{Ti}$ ) this difference is not at all noticeable. We inferred that the (b) self-consistent scheme is the best choice when dealing with the K-edge. Our general conclusion is that the self-consistency brings no major change at high energies, as compared to the non-self-consistent calculations. In some cases ( $\mathrm{Fe}$ and $\mathrm{Ni}$ ) one can see a shift in the position of the low energy peaks. This behaviour confirms our assertion that the self-consistent effects occur mostly in the vicinity of the Fermi level.

We note that, in the case of transition metals, one can allow to run the self-consistent calculation on a cluster of a lesser radius than the one for the absorption calculation. A cluster containing two atomic shells surrounding the absorber is large enough to render reasonable results for the self-consistent part of the calculation. We tested this feature on the transition metals and we observed that the absorption calculation run for a given radius (of the order of $5 \AA$ or more) is not sensible on the previous self-consistent calculation step provided the latter contains two or more atomic shells.

In the following paragraph we shall discuss the results we obtained on $\mathrm{CaO}$ and $\mathrm{BN}$, some prototypical compounds where non-spherical effects are important. In both cases, we compared the self-consistent and the non-self-consistent results (figures 9 and 10) with the experiment [11, 12]. Among the self-consistent calculations, schemes (b) and (c) give similar results and we chose to show the former one. For these particular materials, the calculated Fermi level is not the same with the cutoff we used for the convolution (for the quantitative information, see the captions of figures 9 and 10). We explain this inadequacy by the fact that our assumption of sphericity (the MT approximation) prevents us counting all the electrons, provided that we are not allowed to exceed an upper limit of the overlap of the $R_{j}$ integration spheres. This energy shift does not usually appear whether the compound is a compact structure, as non-spherical effects are negligible, but may be significant if one deals with a sparse structure, like $\mathrm{BN}$ or $\mathrm{CaO}$. We note that it is still the self-consistent calculation which gives a more reasonable result with respect to the Fermi level evaluation.

In the case of $\mathrm{BN}$, one can see practically no difference between the self-consistent and the non-self-consistent calculations, whether the last iteration was performed in the FDM or in the MST-MT frame. Moreover, the agreement with the experiment is obviously better in the case of the FDM calculations. One concludes that it is crucial for this particular material to be treated within a full potential method (FDM), whereas the fact that the calculation is self-consistent or not (we recall that the self-consistency was implemented in the MST frame, due to computational limits) does not make any difference.
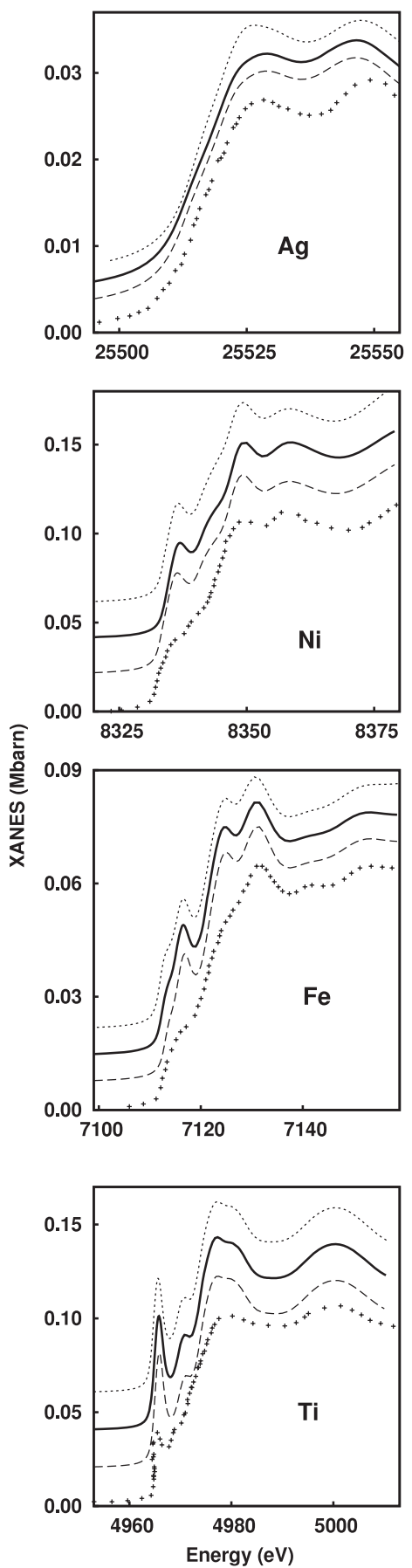

Figure 8. Calculations-non-SCF (long dashes), SCF-(b) (solid line), SCF - (c) (short dashes) and experimental data (with dots) for some transition elements. All calculations were performed on a $7 \AA$ cluster. The absorption units match the calculation and the experimental data is scaled for comparison. Self-consistency does not bring significant changes; nevertheless it allows a judicious estimation of the Fermi level. Among the several alternatives, scheme (b) is to be preferred.

As far as $\mathrm{CaO}$ is concerned, one notices an amelioration provided by the self-consistent calculations, in both the MSTMT and FDM cases. Nevertheless we are still unable to get a perfect agreement for the modulations in the range 4060-4070 eV. One can see (figure 9) a better agreement in the case of a non-self-consistent FDM absorption calculation 


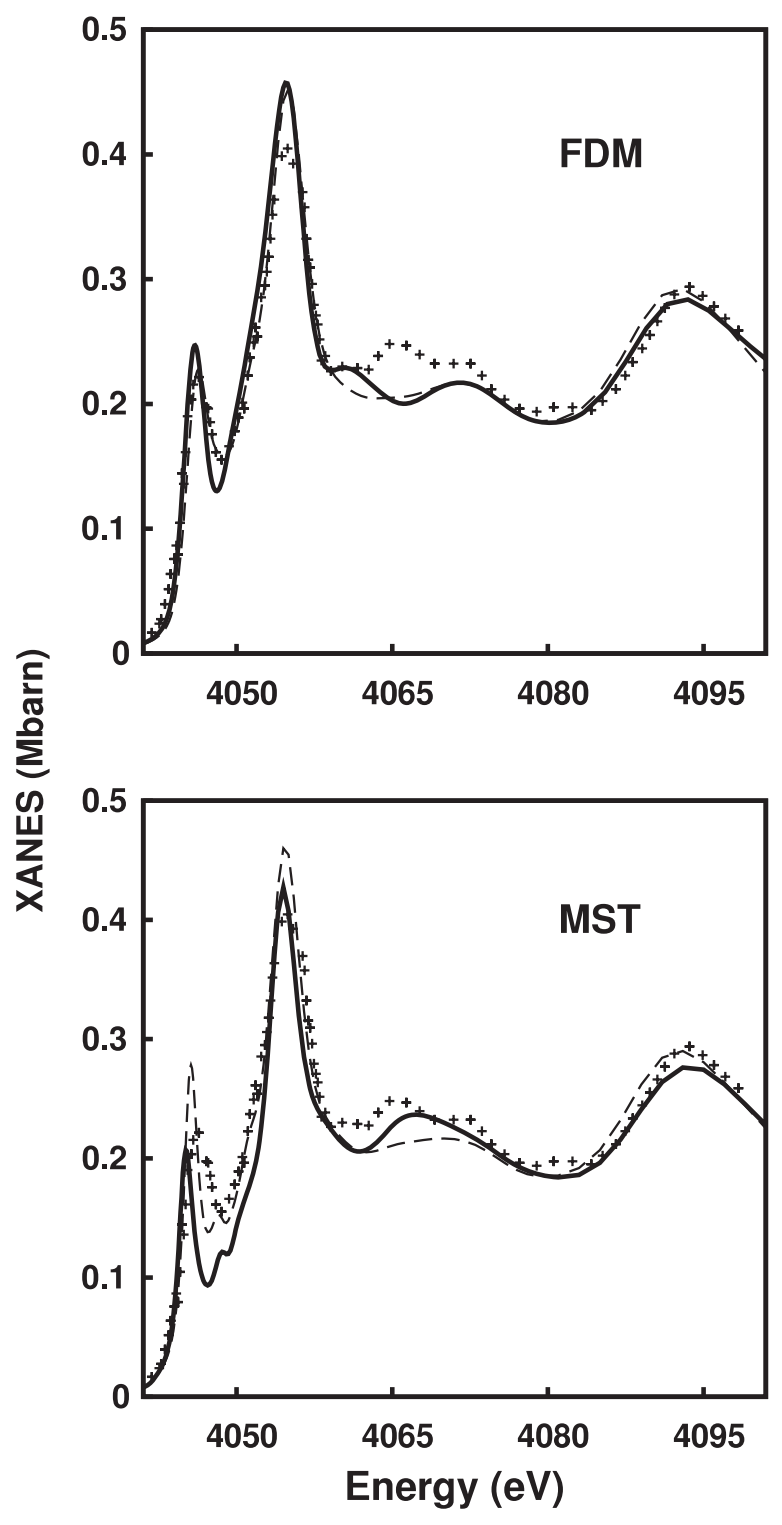

Figure 9. Comparison between the self-consistent (solid line) and non-self-consistent (dashed line) calculations with the experimental data [12] (with dots), for calcium oxide $\mathrm{CaO}$, with the absorption calculation performed in the FDM (above) and in the MST-MT (below). We used a $6.9 \AA$ cluster, i.e. 93 atoms. The absorption units match the calculation and the experimental data is scaled for comparison. The calculated Fermi level $(4037 \mathrm{eV}$ for the non-self-consistent calculation and $4039.5 \mathrm{eV}$ for the self-consistent one) is different from the cutoff we used for the convolution $(4042 \mathrm{eV})$

than for the self-consistent MST-MT one. This leads us to the conclusion that taking into account non-spherical effects is more important than the amelioration given by the selfconsistent procedure, as implemented in the FDMNES (i.e. in the spherical potential approximation).

\section{Conclusions}

One concludes that at the K-edge the effects of self-consistency (in terms of the positions of the peaks) are restrained to the pre-

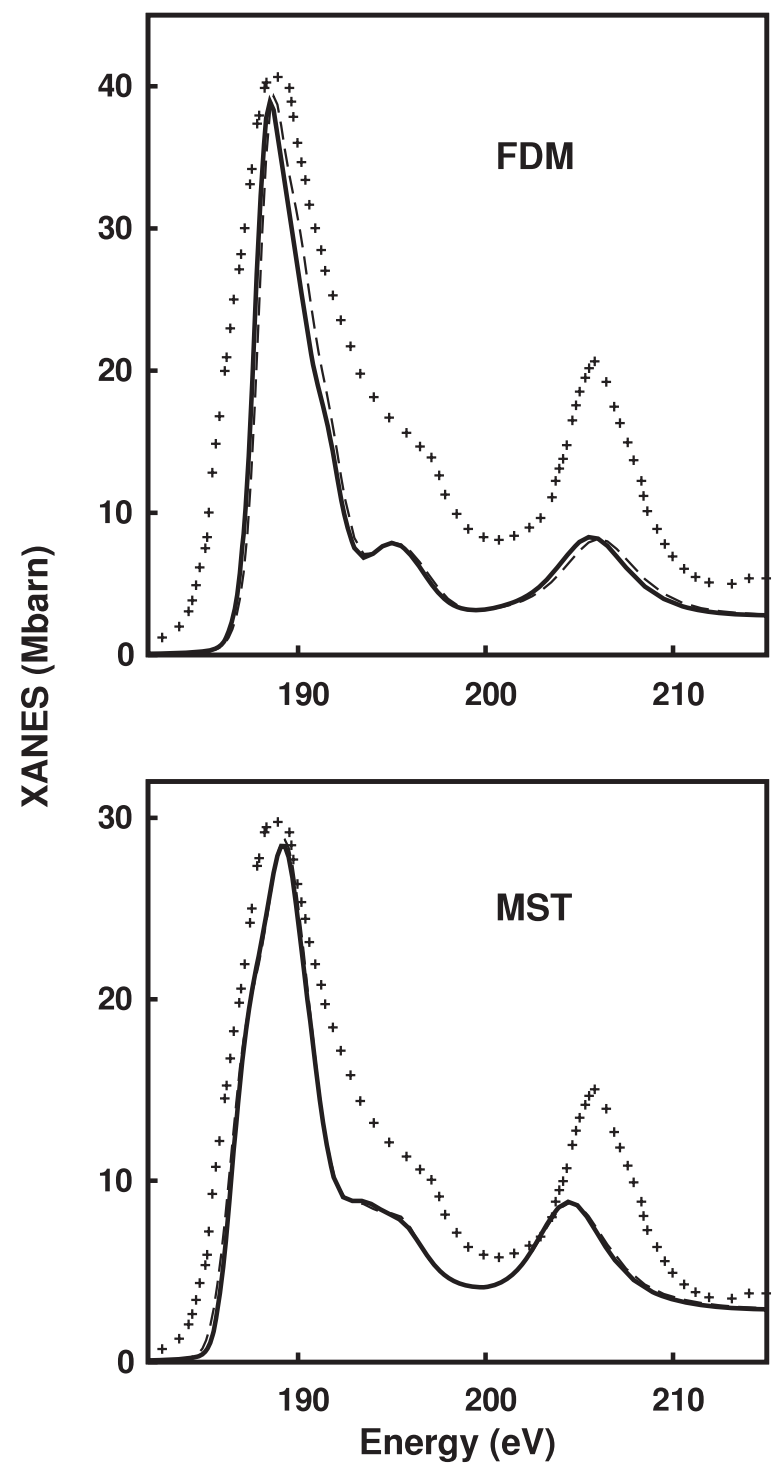

Figure 10. Comparison between the self-consistent (solid line) and non-self-consistent (dashed line) calculations with the experimental data [11] (with dots), for boron nitrate BN, with the absorption calculation performed in the FDM (above) and in the MST-MT (below). We used a $4.7 \AA$ cluster, i.e. 87 atoms. The absorption units match the calculation and the experimental data is scaled for comparison. The calculated Fermi level $(178.2 \mathrm{eV}$ for the non-self-consistent calculation and $179 \mathrm{eV}$ for the self-consistent one) is different from the cutoff we used for the convolution $(186 \mathrm{eV})$.

edge structures, close to the Fermi level. For certain materials, a slight redistribution of the intensities can be noticed at high energies. In the cases where non-spherical effects are expected, performing calculations with a full potential method is more important than the benefits one gets by means of the self-consistency in the MST-MT frame. The value of the Fermi level is very useful information one gets from a selfconsistent calculation, as it is essential for the identification of the occupied states and thus for the comparison with the experimental spectra. This cutoff of the spectra is correctly estimated for the compact structures, but is liable to adjustment in the case of materials where non-spherical effects are 
important. We have proved that the self-consistency brings practically no change for the compact structures, in which case one should be content to run the self-consistent part of the calculation at a lower radius than the one used for the absorption part. Oxides require that one uses the same cluster sizes for the two stages of the calculation for the purpose of improving the description of pre-edge structures.

\section{Acknowledgments}

The authors are very grateful to C R Natoli and C Brouder for fruitful discussions. This work has been done with the support of the LightNet European Network.

\section{References}

[1] de Groot F 2005 Multiple effects in x-ray spectroscopy Coord. Chem. Rev. 249 31-63

[2] Joly Y 2001 X-ray absorption near edge structure calculations beyond the muffin-tin approximation Phys. Rev. B 63 125120-9

[3] Natoli C R, Misemer D K, Doniach S and Kutzler F W 1980 First-principles calculation of $\mathrm{x}$-ray absorption-edge structure in molecular clusters Phys. Rev. A 22 1104-8

[4] Benfatto M, Della Longa S and Natoli C R 2003 The MXAN procedure: a new method for analysing the XANES spectra of metalloproteins to obtain structural quantitative information J. Synchrotron Radiat. 10 51-7

[5] Ankudinov A L, Ravel B, Rehr J J and Conradson S D 1998 Real space multiple scattering calculation of XANES Phys. Rev. B 587565

[6] Cabaret D, Gaudry E, Taillefumier M, Sainctavit Ph and Mauri F 2005 XANES calculation with an efficient non muffin-tin method: application to the angular dependence of the al k-edge in corundum Phys. Scr. T 115 131-3

[7] Blaha P, Schwarz K, Madsen G, Kvasnicka D and Luitz J 2001 An Augmented Plane Wave + Local Orbital Program for Calculating Crystal Properties TU Wien

[8] Ebert H 2000 Electronic Structure and Physical Properties of Solids vol 535 (Berlin: Springer) chapter (Fully Relativistic Band Structure Calculations for Magnetic Solids Formalism and Application) p 191

[9] Olevano V, Reining L and Sottile F http://theory.polytechnique. $\mathrm{fr} / \mathrm{codes} / \mathrm{dp}$
[10] Kruger P and Natoli C R 2005 Multi-channel multiple scattering theory for XAFS application to the 12, 3 edges of Ca Phys. Scr. T 115 146-8

[11] Ankudinov A L, Ravel B, Rehr J J and Conradson S D 1998 Real-space multiple-scattering calculation and interpretation of x-ray-absorption near-edge structure Phys. Rev. B $\mathbf{5 8} 7565-76$

[12] Modrow H, Bucher S, Rehr J J and Ankudinov A L 2003 Calculation and interpretation of k-shell $\mathrm{x}$-ray absorption near-edge structure of transition metal oxides Phys. Rev. B 67035123

[13] Blume M 1994 Resonant Anomalous X-Ray Scattering (Amsterdam: Elsevier) p 495

[14] Perdew J P and Wang Y 1992 Accurate and simple analytic representation of the electron-gas correlation energy Phys. Rev. B 45 13244-9

[15] Hedin L and Lundqvist S 1971 Explicit local exchange-correlation potentials J. Phys. C: Solid State Phys. 42064

[16] Von Barth I U and Hedin L 1972 A local exchange-correlation potential for the spin polarized case J. Phys. C: Solid State Phys. 51629

[17] Krause M O and Oliver J H 1979 Natural widths of atomic k and 1 levels, $\mathrm{k}$ alpha $\mathrm{x}$-ray lines and several kll auger lines J. Phys. Chem. 8 329-38

[18] Seah M P and Dench W A 1978 NPL Report Chem 82 National Physics Laboratory, Div. of Chemical Standards, Teddington, Middlesex

[19] Brouder Ch and Kappler J P 1997 Magnetism and Synchrotron Radiation (Les Ulis: Les Editions de Physiques) pp 33-54

[20] Natoli C R, Benfatto M and Doniach S 1986 Use of general potentials in multiple-scattering theory Phys. Rev. A 344682

[21] von Barth U and Grossmann G 1982 Dynamical effects in x-ray spectra and the final-state rule Phys. Rev. B 25 5150-79

[22] Privalov T, Gel'mukhanov F and Ågren H 2001 Role of relaxation and time-dependent formation of $\mathrm{x}$-ray spectra Phys. Rev. B 64165115

[23] Kohn W and Sham L J 1965 Self consistent equations including exchange and correlation effects Phys. Rev. A $1401133-8$

[24] Poumellec B, Cortes R, Tourillon G and Berthon J 1991 Angular dependence of the Ti k edge in rutile $\mathrm{TiO}_{2}$ Phys. Status Solidi b 164 319-26

[25] Moruzzi V L, Janak J F and Williams A R 1978 Calculated Electronic Properties of Metals (Oxford: Pergamon)

[26] EXAFS Materials 871 El Cerro Blvd., Danville, CA 94526 USA 